\title{
Desterritorializaciones educativas para la Universidad de la Sociedad del Conocimiento
}

\author{
Andrade,Raiza* \\ Méndez, Raizabel ${ }^{* *}$ \\ Martínez, Don Rodrigo ${ }^{* * *}$
}

\begin{abstract}
Resumen: Tres gnoseologías para la Universidad de la Sociedad del Conocimiento. Una, parte de considerar que la palabra escolar y la palabra de la existencia, preñadas de viejos paradigmas, se han transformado en silencio muerto, no son más silencio creativo, por ello, jugar a desempalabrar(nos) caórdicamente emerge como uno de los caminos para aprender a desaprender y desdecir certezas desaprendiendo(nos). Otra, postula derribar los muros disciplinares del aula para Rizomáticamente Co-entrelazar Aprendizajes (ARCA) cogno-vivenciales entre docentes-cartógrafos y arqueontes-creactores en una Aula para/de/desde la Complejidad. En la tercera, el cognoscimiento como praxis de la Complejidad re-liga, se articula/des-articula y re-articula espiralíticamente, caordisciente y cogno-conscientemente: del orden - al caos - al orden, del decir y del disentir al paradojar y al metaforar, en una construcción y de-construcción de sentidos de carácter continuo y permanente. Estas estrategias multi-inter-trans-epistémicas de aprendizajes/desaprendizajes/re-aprendizajes cogno-meta-caordiscientes son concebidas como modos de proceder, de aprender y de con-vivir, onto-trans-epistémicamente.

Palabras clave: desempalabramiento caórdico, aula rizomática, onto-transepistemología, complejidad
\end{abstract}

\section{Educational deterritorialization for the Society of Knowledge University}

\begin{abstract}
Three gnoseologies for the Society of Knowledge University: the first one starts with the consideration that the school word and the existence's word, both loaded with old paradigms, have transformed into dead silence and are no longer creative silence. Therefore, to play chaordic deconceptualization emerges as one of the ways to learn to unlearn; discarding certainties by the act of unlearning(ourselves). The second one postulates tearing down the disciplinary walls of the classroom in order to create an Area in which, Rhizomatically, Cognoexistential and experiential learning Acquisition (ARCA) is co-intertwined between cartographer-teachers and creactors-arqueontes in a classroom of/from/for Complexity. The third one states that cogknowledge as a praxis of Complexity re-
\end{abstract}

\footnotetext{
* Universidad de Los Andes (ULA), Mérida, Venezuela. Email: raizaandrade@yahoo.com

** Universidad de Los Andes (ULA), Mérida, Venezuela. Email: raiza99@yahoo.com

*** Universidad de Los Andes (ULA), Mérida, Venezuela. Email: donrmarta@yahoo.com
} 
links, articulates/disarticulates/rearticulates in a chaordically spiralitically cognoconsciously fashion, going from order to chaos to order, from stating to dissenting while paradoxing or metaphoring, in a continuous and permanent construction/ deconstruction of meanings. Such multi-inter-trans-epistemic strategies for cognometa-chaordically learning/unlearning/relearning are conceived as ways to carry oneself, to learn and to co-exist in an onto-trans-epistemic way.

Key words: chaordic unwording, rhizomatic classroom, onto-transepistemology, complexity

\section{Desterritorializacões educativas pra Universidade da Sociedade do Conhecimento}

Resumo: Três gnoseologias pra Universidade da Sociedade do Conhecimento. A primera, parte que o palavra escolar e a palavra de vida, cheio de velhos paradigmas, transformaram-se em silêncio absoluto, o silêncio que eles não são mais silêncio criativo, portanto, jogar a desempalabrar-nós caórdicamente emerge como uma das formas de aprender a desaprender e voltar em certezas desaprender-nós. Outra postula demolir as paredes disciplinarias da sala de aula para Rizosomaticamente Co-entrelazar Aprendizagem (ARCA) cogno-experienciales entre professores-cartógrafos e arqueontes-creactores em uma sala de aula para / de / desde a Complexidade. Na terceira, o reconhecível como práxis da Complexidade é re-ligado, organizado / un-estruturado e re-articulado espiralíticamente, caordisciente e cogno-conscientemente: da ordem - ao caos - a ordem, e da dissidência a o paradoxo e a metáfora, em uma construção e desconstrução de significados de caráter contínuo e permanente. Estas estratégias multi-inter-trans-aprendizagem epistêmico / des- aprendizagem / re-aprendizagemmeta- cogno-caordiscientes cogno são concebidas como formas de proceder, para aprender e para con-viver, trans-onto-epistemicamente.

Palavras-chave: desempalabramiento caórdico, aula rizomatica, onto-transepistemologia, complexidade

Recibido: 15.01.2010

Aceptado: 07.02.2010

$* * *$

\section{Abrevando en fuentes transdisciplinarias}

No hay método, no hay receta, sólo una larga preparación

G. Deleuze

La perspectiva de abordar el aprendizaje de manera pertinente con las nuevas realidades se entreteje con lo que se ha dado en llamar la Sociedad del Conocimiento (concepto que surge a finales de los sesenta en "The Age of Discontinuity", Drucker, 1968), porque en ella se desarrollan capacidades para generar, apropiar y utilizar conocimiento y dirigirlo hacia la atención de necesidades que coadyuven en la construcción del futuro, convirtiendo la creación y la transferencia del conocimiento en herramientas fundamentales para el logro de objetivos transformadores. 
La Sociedad del Conocimiento es una sociedad marcada por un aprendizaje permanente que exige la revisión y adecuación constante de los diferentes actores e instituciones, para asumir y orientar el cambio y para desarrollar modelos que transformen la información - que se produce de manera exponencial y pluridimensional - en conocimiento útil con efectividad social. A ella le será esencial el aprendizaje a lo largo de la vida ("lifelong learning”) (Longworth citado en Fainholc, 2005), que significa oportunidades educativas diseñadas de modo flexible y personalizado, entendidas como acompañamiento a lo largo de la vida personal y colectiva, como procesos de aprendizajes globales, integrales, reales y virtuales.

La Sociedad de la Información y el Conocimiento como conceptos emergen entonces, caracterizándose en su esencia como un nuevo paradigma cultural de aprendizaje permanente en el cual las personas se forman según necesidades diferentes de aprendizajes diversos en el marco de la Complejidad; aprendizajes que exigen desaprendizajes continuos e innovaciones en la manera de abordarlos. Cabe la pregunta que vincula a la Universidad en este contexto ¿Qué está haciendo la Universidad para introducir cambios que le otorguen pertinencia a su acción educativa en ambientes complejos? ¿Los profesores y los profesionales egresados de las aulas universitarias están preparados para enfrentar ambientes de incertidumbre, de coexistencia de lógicas diversas, para abordar la ausencia de certezas y la acelerada obsolescencia del conocimiento? ¿Los investigadores de la Complejidad están respondiendo a la necesidad de aportar un lenguaje y una praxis social que permita profundizar en experiencias concretas sus postulados teóricos, conceptuales y paradigmáticos?

Imaginar nuevos territorios entrelazados y transdisciplinares para una praxis de la Complejidad que impregne la Educación Superior y permita transformaciones de las instituciones universitarias para hacerlas pertinentes con la Sociedad del Conocimiento y el Pensamiento Complejo (Morin, 1997) resulta indispensable para abordar la co-construcción de las nuevas realidades.

La transdisciplinariedad que subyace en toda praxis de la Complejidad, se corresponde, en el sentido que le imprime Flores (2006), con una decisión epistemológica que implica una transposición de la idea clásica de conocimiento como contenidos y aprehensión de objetos, por aquélla que apuesta a una radicalización del saber como verbo y no más como sustantivo. De esta forma la oposición entre sujeto y objeto quedaría sobrepasada por una concepción de sujeto que conoce siempre en un mundo ya dado del cual él es su principal intérprete. Así "la textura de lo real se perfila desde la subjetividad”. De esta manera, la transdisciplinariedad no se limita a una simple cuestión metodológica de tematización de ciertos contenidos afines sino que se sustenta en la convicción de que el conocimiento siempre es parte de una red de enlaces sistémicos, que los sujetos interpretamos creamos y compartimos bajo ciertos criterios de convención, aportados por el ejercicio científico, y la adhesión a determinados paradigmas de comprensión (Ibid.). 
Se comparte con Najmanovich (2006) que uno de los aportes fundamentales que nos da la Complejidad es el habilitar nuevos interrogantes, gestar miradas diferentes sobre el mundo que nos incluye. La Complejidad lleva implícita la posibilidad de una multiplicidad de modulaciones temporales, entre las que destaca la dinámica en forma de bucle, responsable de la mayoría de las paradojas, de un abordaje que enfrenta el desafío de gestar una concepción del conocimiento en que la teoría no esté divorciada de la praxis, los afectos de los pensamientos, ni el sujeto del ecosistema.

Vista desde esta perspectiva la Complejidad ofrece la oportunidad de insuflar sentido en nuestras prácticas sociales, en nuestro modo de conocer, de legitimar y compartir el saber, es decir, de enriquecer nuestros territorios existenciales en múltiples dimensiones. Muchas experiencias que la mentalidad y la sensibilidad dicotómicas habían desvalorizado, invisibilizado, e incluso rechazado o negado, podrán ahora formar parte de un paisaje vital que no se construye a partir de exclusiones a-priori. (Ibid.).

Desde el año 2002, como parte de las investigaciones y actividades académicas que realizan los autores en la Universidad de Los Andes (ULA), Mérida, Venezuela, éstos han venido vinculando teoría y praxis de la Complejidad, lo que ha conducido a la formulación de tres gnoseologías para la educación Superior en la Sociedad del Conocimiento.

Una $^{1}$ de ellas parte de la necesidad de aprender a desaprender y desdecir certezas desaprendiendo(nos). Considera que las palabras, preñadas de viejos paradigmas, se han transformado en silencio muerto, no son más silencio creativo. Postula estrategias para desaprender, entre las que se encuentra el jugar a desempalabrar(nos) caórdicamente como uno de los caminos que facilitan el desaprendizaje.

La segunda ${ }^{2}$ gnoseología plantea la necesidad de rizomáticamente co-entrelazar aprendizajes en un modelo de aula para/de/con la Complejidad denominado ARCA, en la cual los muros disciplinares del aula-tradicional se derriban para facilitar la confluencia de docentes-cartógrafos y arqueontes que en el mismo espacio cognovivencial, se abren a los nuevos contextos de aprendizajes no lineales, a entrelazamientos de significados dialógicos, borrosos e inciertos.

En la tercera ${ }^{3}$, la simbiosis entre teoría y práctica se expresa en estrategias multi-inter-trans-epistémicas de aprendizajes/des-aprendizajes/re-

\footnotetext{
${ }^{1}$ Aprender a desaprender. Juegos dialógicos de desaprendizaje cognoconsciente, Tesis Doctoral ,UNIEDPA, Panamá, 2002, de Raiza Andrade

${ }^{2}$ Principios gnoseológicos del co-entrelazamiento de aprendizajes en el contexto de la complejidad. Hacia un Aula Rizomática Co-entrelazadora de Aprendizajes (ARCA) desde una perspectiva Cognovivencial, Tesis Doctoral, UNIEDPA, Panamá, 2009, de Raizabel Méndez.

${ }^{3}$ Onto-trans-epistemología compleja de la creactividad. Co-osadías caordiscientes para "darse a luz” creactores, Tesis Doctoral, UNIEDPA, Panamá, 2009, de Don Rodrigo Martínez.
} 
aprendizajes cogno-meta-caordiscientes, concebidas como modos de proceder, de aprender y de con-vivir, onto-trans-epistémicamente. Estas estrategias configuran una Onto-trans-epistemología de la Creactividad en la que el cognoscimiento, como praxis de la Complejidad y el Pensamiento Complejo, re-liga, se articula/des-articula y re-articula espiralíticamente, caordisciente y cogno-conscientemente: del orden - al caos - al orden, del decir y del disentir al paradojar y al metaforar, en una construcción y de-construcción de sentidos de carácter continuo y permanente.

\title{
Desempalabramientos caórdicos para aprender a desaprender ${ }^{4}$
}

\author{
"La palabra es a la vez maravillosa y terrible" \\ B. Cyrulnik \\ “Toda palabra es un prejuicio"
}

F. Nietzsche

En el Nuevo Testamento se le atribuye a Juan El Bautista la afirmación de que "En el principio era el Verbo y el Verbo era con Dios, y el Verbo era Dios” (1:1,14). Así, la palabra, el logos y el habla quedaron imbricados. Esos sonidos que comunican un significado, encarnan una memoria, unas normas, una costumbre y hacen de la lengua "un cuerpo" (Derrida, 2001:56). Un cuerpo tatuado de decires a los que atribuimos significados personales, significados culturales y significados paradigmáticos.

Dilthey (1960) se refiere a la palabra como representación y como relación; interpreta e incorpora lo particular de cada una cuya significación llega a ser aprehendida; su "sentido implica lo vivido y lo posible de ser vivido, experiencias propias tanto como ajenas, tradición como presente" (:55). Con las palabras empleadas por esa inteligencia consciente que nos diferencia de la inteligencia animal, Morin, en su diálogo con Cyrulnik (2005), afirma que creamos una "noosfera, es decir una esfera de productos de nuestras mentes” (:25), construimos ideas que ejercen una mediación sobre la realidad pero que también la encubren.

Las palabras se han vaciado de sentido y requerimos "cartografiar, incluso, nuevos parajes” (Deleuze y Guattari,1994:10), deslindar territo-

\footnotetext{
${ }^{4}$ Desde el año 2002 hasta la fecha, la autora viene experimentando con juegos dialógicos cognoconscientes para aprender a desaprender realizados con participantes del Diplomado Internacional en Creatividad y Liderazgo (DICYL), de la Universidad de Los Andes, Mérida, Venezuela. Parte de los resultados de esa experiencia se encuentran incorporados en la Tesis Doctoral en Educación de Raiza Andrade y forman parte del Proyecto de Investigación $\mathrm{N}^{\circ}$ A-730-09-04-B financiado por el Consejo de Desarrollo Científico, Humanístico y Tecnológico (CDCHT), de la mima Universidad.
} 
rios, desterritorializar significados que han perdido la capacidad de nombrar. Ir al encuentro de aquellas palabras que nos permitan conocer al mundo como "mi voluntad y mi representación”, (Schopenhauer, 1818), conscientes, a la vez, de su total "incognoscibilidad"(Ibid.); aspirar a "re-decirnos”, a alcanzar una nueva "relación con el habla” (Heidegger, 1987:242) que nos permita una re-conceptualización simbólica, referencial y conceptual en sintonía polifónica con la Complejidad y con "todo decir esencial [que] es retorno para prestar oído a esta mutua pertenencia del decir y ser, palabra y cosa" (Ibid.:213). El ser del hombre reside en el habla como diría Heidegger y el habla es palabra y silencios entretejidos.

Observamos el mundo y en el camino de comprenderlo (nos), lo/nos empalabramos. Pero esas palabras se encuentran preñadas de sentidos que le imprimen los paradigmas dominantes. Decía Foucault (1979) que hasta el Renacimiento existía una identidad entre las palabras y la realidad hasta que surgió Descartes y la palabra pasó a ser representación. Esa visión cartesiana ha permeado toda nuestra manera de mirar y comprender al mundo y a nosotros mismos, las palabras han mutado en tatuajes que condicionan nuestras formas de conocer. Planteábamos que

Los paradigmas se encuentran sumergidos en los conceptos. La construcción del conocimiento y la comprensión del universo dependen de la palabra que nombra, que define. Empalabramos el mundo y a partir de la palabra reconocemos la realidad que nos contiene. El paradigma de la Complejidad, retoma la consideración de una identidad entre pensamiento, lenguaje, conocimiento y expresión. Se enfrenta [en consecuencia] a la necesidad de renombrar, in-formar, re-formar, de reconocer las nuevas maneras de decir y desdecir esa danza sagrada de las realidades complejas, en la que participan la mente, la materia, la naturaleza, la vida, el cosmos y la red de relaciones de un sistema cada día más globalizado. Aprender a desaprender pasa por reconocer los límites de la palabra, por reconocer, en consciencia, los tatuajes, las improntas que el paradigma cartesiano ha sembrado en nosotros. Las palabras ocupan ahora un lugar al lado de un habla que interroga más que afirma, que dialoga más que sentencia, que se reconoce despojada de verdades absolutas, incompleta, desordenadora, detonadora de realidades infinitas e inimaginadas. (Andrade, 2004:28,29)

En los últimos años hemos jugado, en el marco de la academia, a desempalabrar, a desempalabrarnos, como una lúdica dialógica de cognoconsciencia, como uno de los caminos para aprender a desdecir certezas, desaprendiendo(nos). Aprender a desaprender desde el desempalabramiento de sí, es desaprender aquello de lo que nos hemos apropiado o que se ha apoderado de nosotros para instalarse como lo que "es”, como una "verdad inmutable". 
En las aulas de la Educación Superior resulta necesario aprender a empalabrarnos/des-empalabrarnos/re-empalabrarnos caórdicamente. Ésta es una tarea pendiente para ser llevada a cabo en consciencia cognoconsciente de que, más pronto o más tarde, nos desempalabraremos re-deconstruyendo-nos una y otra vez, constituyendo tal acción, un desafío en este devenir como sujetos de una Complejidad caórdica.

Entendemos los desempalabramientos como caórdicos ${ }^{5}$ dado que se asumen como:

Creativos: lúdicos e imaginados. En la consciencia de la necesidad permanente de re-crear palabras y conceptos que trasciendan aquellos que limitan el decir (nos), que se encasillan en preceptos viejos, que paralizan, condicionan, reducen y limitan el proceso de comprensión de Sí mismo y del Todo.

Amalgamados, en esa heterogeneidad que somos. Desempalabramientos que articulen cadenas de palabras por desempalabrar, cuya referencia se vincula a los procesos de vida y muerte del conocimiento.

Objetantes, en términos de mirar el juego empalabrador de las palabras-conceptos desde una perspectiva crítica. Desacostumbrarnos al uso de las palabras que perdieron su capacidad de nombrar las nuevas realidades.

Resonantes, en tanto desempalabramientos que sirven de espejos para la construcción y deconstrucción de las conexiones que las palabras llevan implícitas.

Desplegados, capaces de explicitarse mostrando y dejando en evidencia los tatuajes de la palabra, las huellas sinópticas y sinápticas condicionadoras de métodos, procesos, actividades, procedimientos que signan el aprender a aprender

Inacabados, y por ello demandan un trabajo permanente de consciencia, de autoreflexión, que facilite la construcción y deconstrucción de significados. El empalabramiento y el desempalabramiento como herramientas detonadoras de cambios en los procesos de aprender a desaprender. Puntos de partida que se hacen instantes continuos para el reinicio de procesos que desvistan las palabras para que sean re-nombradas en un continuum de comprensiones y re-comprensiones, de significaciones y resignificaciones para decirnos y desdecirnos en el camino de aprender a aprender, a desaprender, a re-aprender.

Conjugantes, porque al desempalabrarnos “jugamos-con” esa particular manera en la que hemos aprendido a decirnos y a conocer. "Jugamoscon” la posibilidad de entrelazar significados en los territorios del singular y

\footnotetext{
${ }^{5}$ Concepto, éste último, resignificado para este contexto, tomando como inspiración la definición propuesta por Andrade y Pereira (2006) en relación a las “Fárbices Caórdicas”.
} 
del plural, del yo y del ellos, del tú y el nosotros para desterritorializarnos en consciencia de aquella palabra que ha dejado de decir o que nos limita el decir.

Ontodinámicos, ya que se hacen posibles a partir de motivaciones personales, íntimas, de consciencia. Se asumen desde el reconocimiento de los límites. Muestran los vacíos, las redundancias, las confusiones. Facilitan posibilidades de nuevas comprensiones detonadas a partir de la emergencia de la palabra empalabradora y deslimitante.

Sinórdicos, al emerger de forma inesperada, "sin orden” predefinido. El desempalabramiento ocurre asecuencialmente, de manera sinfónica, simultanea. Su significado se muestra después, cuando miramos los tatuajes que afloran en cada recorrido cargados de sentidos que únicamente el “desempalabrador” es capaz de interpretar.

Desde el año 2002 hemos venido trabajando con imaginaciones creativas que detonan desempalabramientos caórdicos las cuales se han aplicado a los participantes del Diplomado Internacional en Creatividad y Liderazgo (DICYL) y a grupos ad hoc conformados para una praxis de la Complejidad desde nuestra línea de investigación en torno al aprender a desaprender. Numerosas han sido las respuestas de ese devestirse de palabras para encontrar los límites y los condicionamientos impuestos a nuestros propios procesos del conocer. Uno de esos testimonios de la profesora Regina Drumond (Brasil) participante del ejercicio en la IV edición del año 2005 refleja el impacto de la dinámica lúdica de desempalabramiento que hemos diseñado. En sus palabras testimoniales se recoge la experiencia:

Se me plantea el reto: Estamos tatuados de palabras, en la vida cotidiana empalabramos el mundo. Las ideas no son únicamente aquellas que poseo, son además las que me poseen... ¿Estaré abierta a cambiar? ¿Seré capaz de sorprenderme? Palabras, símbolos, pensamientos, son apenas límites, pues todas las veces que pensamos o usamos una palabra o norma, estamos creando límites. En el lenguaje dualístico, las palabras nos limitan y la realidad se encuentra más allá de los límites, normas, formas, palabras y pensamientos. Somos renuentes a abandonar estos límites en la percepción sin límites, en la conciencia de la unidad, la noción del yo se expande de manera de incluir todo lo que antes era considerado no yo. A través de la magia de las palabras, palabras que son como una espada de dos filos, donde lo que vive por la espada perece también por la espada, me descubro.

Nuestras palabras, símbolos, señales, pensamientos e ideas son apenas mapas de realidades, porque el mapa no es el territorio, la palabra mapa no mata mi sed, pero vivo en un mundo de mapas y palabras como si fuese el mundo real. Nuestra conciencia cotidiana es una isla insignificante cercada por un vasto océano de conciencia inexplorada, cuyas ondas baten continuamen- 
te sobre la barrera de arrecifes de nuestra conciencia normal hasta que rompa las barreras invadiendo nuestra percepción insular con un conocimiento de un dominio enorme, inexplorado, más real, de la conciencia de un nuevo mundo. Mi línea de límites puede ser rediseñada, desaprendida, reaprendida para encontrar nuevos territorios y si se pierden estos límites, al identificarlo con el todo armonioso, ahí deja de existir lo exteriorinterior entre el no-yo y el yo ¿Será que estoy preparada para salir de esta fase de ser repetidor, de reproducir lo que los otros dicen, de imitar, para la fase de ser yo, de concientizar mis tatuajes y desempalabrar, para tener mayor conciencia de mis bloqueos, flaquezas, límites y dar nuevos saltos? Descubro, con este ejercicio, palabras inimaginadas escondidas en mi cuerpo.

Vivir es afinar el instrumento de adentro para afuera y de afuera para adentro. Aprender a desaprender significa salir de las estructuras mecánicas, cerradas, y estar abierto a diferentes formas de mirar el mundo usando un caleidoscopio de imágenes que cambian a cada segundo, reconociendo que el mapa no es el territorio. Ese entorno comienza dentro de nosotros, en la mesa redonda del Yo. Gracias.

\section{El Aula Rizomática Co-entrelazadora de Aprendizajes (ARCA) ${ }^{6}$}

Hay dos modos de conciencia: una es luz, y otra paciencia.

Una estriba en alumbrar un poquito el hondo mar; otra, en hacer penitencia con caña o red, y esperar el pez, como pescador.

Dime tú: ¿Cuál es mejor?

¿Conciencia de visionario que mira en el hondo acuario peces vivos, fugitivos, que no se pueden pescar,

o esa maldita faena de ir arrojando a la arena, muertos, los peces del mar?

A. Machado

${ }^{6}$ Desde el año 2005 hasta la fecha, la autora viene experimentando con diversas modalidades de ARCA en grupos de estudiantes de pre-grado de la Universidad de Los An- 
La praxis de la Complejidad en el contexto de la Educación Superior demanda el desmontaje de los muros disciplinares que caracterizan al aula tradicional para construir y deconstruir espacios que rizomáticamente permitan, faciliten y detonen procesos de coentrelazamiento de aprendizajes. El modelo propuesto, que hemos denominado ARCA, constituye un territorio de experiencias cognovivenciales. En el mismo, el "educador" se ha transformado en un detonador-cartógrafo de rutas entrelazadas de aprendizajes, por su parte el "aprendiz" ha devenido en arqueonte, entendido como un navegante co-constructor y co-entrelazador de aprendizajes, ambos confluyendo en un aula pensada para/desde/en/de la Complejidad.

Ante la emergencia de la Complejidad entendida como una transformación global de nuestra forma de experimentar el mundo, de coconstruirlo en las interacciones, de producir y de validar conocimientos (Najmanovich, 2006), aprendizajes y existencia, resulta inoperante el modelo arbóreo de enseñanza basado en la separación de saberes, en las disciplinas entendidas como realidades-estancos, en principios tales como: naturaleza única, regiones de dominios, fronteras, jerarquía; conocimientos orientados hacia la producción en serie de profesionales que se acercan de una manera fragmentada a la realidad y al conocimiento; al espacio y al tiempo de su ejercicio profesional. En consecuencia, la Complejidad y su referente educativo imbricado en desde y para el Pensamiento Complejo, requiere del modelaje de nuevas experiencias de aula orientadas al entrelazamiento de conocimientos y vivencias que trasciendan el espacio disciplinar.

Para pensar el "Aula de la Complejidad" se requiere de nuevos constructos que aborden la redefinición del aula tradicional desde la perspectiva de la Sociedad del Conocimiento, desde la imposibilidad de entender al Ser separado del Hacer, del Convivir y del Conocer, en el sentido planteado por Delors (1996) y desde la perspectiva del co-entrelazamiento de aprendizajes, territorios entendidos desde una concepción rizomática para el abordaje del conocimiento. Un pensamiento generador que permita abarcar la realidad en sus múltiples dimensiones, asumirla como un "bucle de recursividades” lo que, según Morin (1997), implica la ruptura de la causalidad lineal; un proceso “... en el cual los productos y los efectos [sean], al mismo tiempo, causas y productores de aquello que los produce” (:106).

Al aplicar los postulados rizomáticos (Deleuze y Guattari, 2003) al “Aula de la Complejidad” puede garantizarse una proliferación de

des, Mérida, Venezuela, organizados de manera ad hoc para la enseñanza de la Propiedad Intelectual. Parte de los resultados de esta experiencia se encuentran incorporados en la Tesis Doctoral en Educación de Raizabel Méndez y forman parte del Proyecto de Investigación $\mathrm{N}^{\circ} \mathrm{D}-380-08-04-\mathrm{B}$ financiado por el CDCHT de la Universidad de Los Andes. 
saberes apoyados en la creatividad, en el tránsito crítico por el conocimiento instituido, en la construcción de nuevas conexiones de sentido, de resignificaciones de contenidos, a partir de entender el saber como un campo abierto a infinitos e impredecibles entrelazamientos, de desarrollar una consciencia del cómo se conoce, en un abordaje del conocimiento desde la desterritorialización de las disciplinas estancos.

El aprendizaje rizomático permite la individuación, favorece el aprendizaje en contextos de incertidumbre, respeta la visión personal en la elección, selección y configuración de significados, facilita una comprensión de lo disciplinar desde una cosmovisión articuladora, favorece la articulación de sistemas educativos abiertos, la autorregulación y el entrelazamiento entre el conocimiento, la profesionalización y la vida. Aprender de manera rizomática permite al individuo generar conciencia de sus capacidades para distinguir, contextualizar, articular, relacionar, resignificar pero también para transgredir, irrumpir, emerger, religar, trascender.

El ARCA es un aula rizomática entendida como el espacio para el desarrollo de procesos de aprendizaje basados en estrategias didácticas centradas en una organización no jerárquica del conocimiento, no lineal. Han sido concebidas a partir de entrelazamientos y emergencias provenientes de necesidades planteadas por los estudiantes, en su contacto con el entorno en el que se desempeñarán como profesionales. Permite las líneas de fuga, abordar aprendizajes no predeterminados en el libre fluir del conocimiento, facilita territorializar y desterritorializar; es un aula de preguntas más que de respuestas, donde las inquietudes derivarán de esas líneas que conducirán hacia el estudio de contenidos, previstos o no, en el programa. Como los contenidos se van planteando a partir de necesidades del grupo, el conocimiento que se imparte se comporta de manera rizomática, ya que los contenidos no necesariamente se ofrecen de manera secuencial articulándose el conocimiento a partir de áreas problema, invirtiendo o trasgrediendo en cualquier sentido, la relación tradicional.

Se hace necesario pensar en un aula de co-aprendizajes para, en, desde, hacia, con la Complejidad, un aula que facilite los entrelazamientos de conocimientos, vivencias y experiencias, que rompa con lo rígido del concepto originario del aula como lugar de encierro, como imagen de espacio estático, un aula del fluir, un espacio para las derivas, para el reencuentro de mundos y visiones de sí, del otro, del Ser, el Hacer, el Conocer y el Convivir. Un "Aula de la Complejidad" como espacio de entrelazamientos de significados que constituya un ejercicio necesario para una praxis de la Complejidad.

El ARCA es un aula autogestante, que se conforma a partir de entrelazamientos de significados compartidos y líneas de fuga que derivan hacia la articulación de nuevos significados. 
En el ARCA, el participante es un arqueonte, un "navegante rizomático de co-entrelazamientos”, un ser autónomo, quien, a partir de conceptos detonadores de inquietudes, irá articulando su realidad particular de conocimiento en la consciencia de sus propias necesidades, de las necesidades actuales o futuras, reales o imaginadas, intuidas, sentidas o presentidas, en el contexto de su quehacer y de su existencia. Este navegante rizomático es un observador que construye, deconstruye y reconstruye su futura realidad profesional, impregnándola con un imaginario de significados.

El camino se construye desde el uno y en la interrelación con el grupo. No se parte de certezas ni se deriva en certezas. La incertidumbre es el camino. El aprendizaje se entiende como un viaje creativo por paisajes laberínticos, que sorprenden sin aviso al aparecer inquietudes transmutadas en líneas de fuga que derivan hacia la profundización y exploración de nuevos contenidos temáticos que se entrelazan en el ARCA.

En tal contexto el docente es un facilitador de entrelazamientos, un detonador-cartógrafo, un tejedor de urdimbres que contribuye con el arqueonte en el diseño de mapas de rutas de aprendizajes transdisciplinarios, un detonador de vivencias desde el Ser, el Hacer, el Conocer y el Convivir, para un entrelazamiento de saberes que dibuja un conocimiento que no nos es ajeno, que se hace y rehace de manera permanente. Ser un detonador-cartógrafo de aprendizajes implica tener consciencia del "trasvasamiento de sentidos" (Barbier,1997), del para qué, del cómo, de los por qué; de lo que se sabe y de lo que se desconoce; de las metáforas y de las islas de certeza; de las preguntas que lo habitan; de la duda ante toda respuesta.

El microespacio del ARCA es el lugar donde se produce ese trasvasamiento de sentidos entre el detonador-cartógrafo de procesos de aprendizajes y los arqueontes. Parte del rol del primero no es solamente el estar consciente de las condiciones en las cuales se produce un entrelazamiento de sentidos, sino el darse cuenta de las complejas condiciones diferenciales de esa producción.

Desde el ARCA, se aprende y se vivencia la imposibilidad de separar el Ser, del Conocer, del Hacer y el Convivir, del pensar y del actuar. El detonador-cartógrafo es un acompañante de procesos, con amplio conocimiento formal e informal sobre las áreas temáticas, formado en la Complejidad y el Pensamiento Complejo, dispuesto a re crear y re crearse, a enredarse, a en-red-darse a/con los arqueontes en la inquietud del día, en la búsqueda de respuestas en conjunto, para la solución de problemas que deriven en nuevas preguntas y nuevas inquietudes; consciente de que cocartografía un mapa de realidades múltiples. 
El detonador-cartógrafo del ARCA, no crea el mapa, está preparado para detonarlo, para leerlo y para contribuir a co-develar sentidos ocultos. El mapa se co-crea y re-crea en la interrelación entre detonadorescartógrafos y arqueontes. Se trata más bien de una especie de "cartografía cognoscente" que se diseña y se hace en el Hacer y el Ser, en el caminar del aprender. Que fomenta la incertidumbre como práctica cotidiana en el ARCA, porque no puede predeterminarse el paisaje del conocimiento ya que los paisajes cobrarán o perderán sentido, desde la mirada de cada arqueonte.

Tenemos la convicción de que la praxis de la Complejidad se va haciendo y deshaciendo, hilando y rehilando en el camino. Emprender multiplicidad de andaduras, explorar una infinita variedad de mapas e itinerarios, que irán emergiendo en el proceso de recreación de la praxis de la Complejidad, es un reto para la educación de hoy que se va haciendo futuro y para el observador, como sujeto complejo entrelazado con lo que observa y con su proceso del conocer.

Compartimos con Sheldrake (2005) que la educación es una "forma de iniciación” (:189), postulamos que en el ARCA el ritual iniciático para el abordaje de la educación desde la Complejidad contemple el aprender a entrelazar, haga de la metáfora del rizoma un lenguaje posible en un proceso de co-construcción del aprendizaje.

Pensar el "Aula de la Complejidad" como un Aula Rizomática Coentrelazadora de Aprendizajes (ARCA), es dar una respuesta, desde el ámbito educativo, a la nueva racionalidad/emocionalidad Compleja, una racionalidad que es dialógica, opta por la transdisciplinariedad, por la borrosidad, la incertidumbre, lo caórdico, los desórdenes creadores, el azar, la heterogeneidad, lo reticular, la libertad de un pensamiento crítico, la confluencia creciente de las disciplinas que deriva en conocimientos producto de tales interacciones, la complementariedad, el entrelazamiento del conocimiento en la realidad.

El ARCA, constituye una respuesta a esa necesidad identificada por Najmanovich (2008) como “... el desafío de construir una nueva gramática centrada en la acción y en la poiesis” (:87), representa una oportunidad transformadora susceptible de recibir modificaciones en el recorrido de su devenir. Constituye, así mismo, una semilla de naturaleza rizomática y posee, por tanto, la virtud de rehacerse y deconstruirse permanentemente y de conquistar otros territorios para cualquier acción relacionada con el conocimiento y el aprendizaje que emerja del azar, de una nueva liana del pensamiento trasvestida de línea de fuga, que mantenga como esencia el recrear el Ser, el Hacer y el Conocer en un Convivir creativo, en/desde/para y con la Complejidad. 


\section{De la Onto-trans-epistemología Compleja de la Creactividad y del “darse a luz" Creactores"}

En esta visión del camino de en medio,

lo que hacemos es lo que sabemos, y el nuestro es sólo uno de muchos mundos posibles. No es un reflejo del mundo, sino un hacerse un mundo, sin ninguna guerra entre el yo y otro.

F. Varela

Cuando situamos nuestro ser en nuestro querer y nuestro querer en la impulsión de que es prolongación, sentimos y comprendemos que la realidad es un crecer perpetuo, una creación que se prosigue sin término.

\section{H. Bergson}

Las concepciones y teorías de la Creatividad han llegado a un límite. Desde el paradigma vigente ésta es vista como herramienta, método, técnica, actitud y las más recientes, como dimensión de las personas; pero no ha sido entendida como la esencia ontológica de lo humano, su semilla-raíz.

La epistemología desarrollada fue primero una intuición que devino en la búsqueda "multi-inter-trans-hermenéutica” de algunos pensadores de las filosofías, las ciencias, las epistemologías, que resonaran, que se hicieran eco, que habitaran en la vecindad del ligar, del interconectar, del entramar, propuesto en esta Onto-trans-epistemología Compleja de la Creactividad.

Es así como el entrelazar pensamientos, ideas, conceptos, como metáforas/paradojas/dilemas argumentativos, nos permitió complementar aquella intuición, buscando rastros, como huellas polifónicas, de algunos de los que han reflexionado sobre tal posibilidad. Ir a la búsqueda de vestigios que nos facilitaran sus signos y señales coincidenciales (como esencias que co inciden), de estelas que abonaran la orquestación firme y flexible, constante y adaptable del pensamiento propuesto, permitiéndonos abrevar de esas fuentes desde la motivación de consolidar aquella intuición y sus multi-significaciones Onto-trans-epistemológicas Complejas, es decir, sumergirnos en el entramado "multi-inter-trans-hermenéutico" que liga al Ser (Creactor-Creactríz) y/con/en sus contextos creactivos.

${ }^{7}$ Desde el año 2003 hasta la fecha, el autor ha llevado a la praxis sus co-osadías caordiscientes para "darse a luz" Creactores en cuatro ediciones del Diplomado Internacional en Creatividad y Liderazgo (DICYL) de la Universidad de Los Andes, Mérida, Venezuela, y en otros grupos organizados de manera ad hoc. Parte de los resultados de esta experiencia se encuentran incorporados en su Tesis Doctoral en Educación. 
Del pensamiento de Guyau (1854-1888) - filósofo francés de la intensificación de la vida, de la emergencia de lo nuevo dentro del proceso evolutivo y la pujanza vital - referimos algunos de sus planteamientos y reflexiones sobre la verdadera autonomía, la cual debe pretender la originalidad individual - y no la uniformidad universal kantiana- una individualidad capaz de crear valores que impriman una certitud personal ante la incertidumbre. Individualidad, valores y originalidad que sirven de base a la propia Libertad (referido en Coppleston, 2004)

De acuerdo con esto la Creactividad, en tanto advenimiento existencial cogno-consciente y multi-dimensional, no pretende ni se afirma en uniformidades universales (Platón, Aristóteles, Descartes, Kant y otros) sino que une, vincula, re-cartografía, telarañiza cosmovisiones, sistemas, disciplinas, caordizándolos desde la Libertad del "Ser-siendo" de los Creactores. Libertad como autonomía cogno-consciente, liberada, “dada a luz" en el camino creactivo-creactivizante. Libertad no como individualidad, separada, sino como proceso caordiscentizador creactivo-complejo co - y trans-epistémico.

Hemos denominado Creactividad, a la esencia ontológica de creatividad-acción y de acción creadora/destructora/re-creadora y creativa en “cogno-meta-consciencia” de Sí, del otro y del entre (espacio/tiempo/circunstancia), que permite la emergencia permanente (mientras dure la "vida") de la multi-dimensionalidad del Todo, del $\operatorname{cosmos}^{8}$, de la vida, multi-fluyendo bio/psico/emo/cogno/socio/espiritualmente de manera trans-entramada en las multi-direcciones de multi-posibilidades de la red del mundo, red que podemos telarañizar y holontotelarañizar para ampliar las comprensiones sistémicas y complejas sobre el multiverso en el que existimos.

El medio por el cual la Onto-trans-epistemología Compleja de la Creactividad trata de perseverar en su Ser, no es el Holismo (reducción al Todo sin ver las partes), ni el Eclecticismo (selección de lo mejor de diversas doctrinas y sistemas), ni el Sincretismo (conciliación de doctrinas diferentes), ni una especie de Sintetismo epistémico (a la manera de compendio que recopila posturas y...); mucho menos pretende ser un extremismo, tampoco un fundamentalismo o radicalismo, negado por la multiplicidad, la pluralidad y la diversidad, pues su Ser no es pendular, en el sentido de ir de un límite a otro manteniéndose en las fronteras, sino que su Ser, en tanto "Ser-siendo", se esfuerza por los tránsitos, procesos, recorridos por el círculo cromático de Verdades y conocimientos, es decir, de las creencias y

\footnotetext{
${ }^{8}$ Incluimos aquí al cosmos porque éste, en tanto palabra que refiere al 'orden universal' de cada cosa en el mundo comprensible, es construido cognitivamente en las representaciones mentales de cada persona desde las distintas 'tradiciones epistémicas' de cada individuo, lengua y cultura (ancestrales, populares, míticas, esotéricas, religiosas, filosóficas, científicas por decir algunas)... pero desde la perspectiva onto-trans-epistémica no se trata de juzgar cual es o no la correcta, sino creactivamente abrirse a la complejidad de visiones y comprensiones con una actitud proactiva entrelazadora multi-inter-trans: disciplinaria/hermenéutica/epistémica.
} 
los valores, de las filosofías, ciencias, religiones, tecnologías, artes, cosmovisiones, saberes populares y tradicionales, entre muchos otros.

Han proliferado numerosas teorías e investigaciones en torno a la Complejidad y el Pensamiento Complejo, sin embargo, continúan teniendo pendientes sus topos de aplicación, su praxis educativa. Por ello, ha resultado esencial proponer la Creactividad como una praxis epistemológica y educativa de la Complejidad y pensar en los Creactores, como seres/actores competentes para crear, re-crear, de-construir/co-construir y co-entrelazar en cogno-meta-consciencia, en/sobre esa realidad compleja, caordiscientizándola y posibilitando sus cogno-vivencias. Caordiscientizar en el sentido de caotizar(se)-ordenar(se)-disentir(se)-sentir(se)cientifizar(se)/des-cientifizar(se)-conscientizar(se).

El/la Creactor/Creactríz es un ser/actor que en esa praxis y desde ella - a un mismo tiempo- es consciente de Sí mismo como un Ser capaz de "re-cogno-ser" sus propias limitaciones y perspectivas personales, abriendo/facilitando/modificando los caminos para aprender/des-aprender/reaprender/de-significar/re-significar/recognoSer para: ser, estar(se), sentir(se), crear(se), tener(se), convivir(se) con las capacidades creativas de soñar(se), imaginar(se), construir/destruir/recrear(se) y estimular(se), en/ con/desde las múltiples competencias, inteligencias y consciencias humanas, y como posibilidad/potencialidad, de manera continua u ocasional, durante su propio devenir "cogno-meta-consciente”, de “darse a luz" Creactores/Creactrices.

"Darse a luz" es un auto-alumbramiento meta-cogno-consciente caordisciente de nosotros mismos en tanto "Ser-siendo"; una liberación creactiva de las atadura invisibles de las muchas “tradiciones” a/en las que estamos sujetados (la mayoría de las veces sin saberlo), para empuñar la Libertad de nuestra propia historia, para "historiarla creactuando" sin radicalismos ni anarquías, sin apegos a ortodoxias ni a dogmatismos hipnotizantes.

Las revelaciones multi-dimensionales del rostro humano, rostro creactivo, se “dan a luz” en la palabra (en sentido ampliado), palabra entendida como posibilidad/potencialidad Onto-trans-epistemológica Compleja Creactiva. Palabras luz que clarifican comprehensiones y sentidos; palabras luz que encienden Vida, Libertad, despertándonos del “adormecimiento/huida”; palabras rostro que nos desnudan desenmascarando las anclas de nuestras "Tradiciones" ciertas y seguras; palabras luz que nos rescatan y desordenándonos nos empoderan, detonando confianza, valentía y autonomía; palabras que iluminan puentes, redes, vínculos para entrelazar mundos y dimensiones. Palabras Luz abiertas al cambio, a la deconstrucción, al desempalabramiento ${ }^{9}$ con el cual crear nuevos rostros, nuevas luces

${ }^{9}$ Desempalabrar: en el sentido de R. Andrade (2005) como juego dialógico de desaprendizaje que permite la desarticulación de los términos encontrando sus significados 
empalabradas de sentidos, comprensiones desde distintas perspectivas con las cuales "multi-inter-trans-hermeneutizarlo" todo, incluso al Ser, a las palabras y a la luz misma, pues los Creactores al "onto-transepistemizar(se)" se “dan a luz" junto a neologismos con los cuales dibujar y colorear nuevos rostros, otros paisajes, la Vida.

Como las epistemologías y los paradigmas nos muestran sólo una cara de la diversidad de posibilidades de comprensión e interpretación del multiverso que habitamos; proponemos pensar en torno a una epistemología que sea múltiple, que se entrelace sin aprensiones/prejuicios/parcialidades/convencionalismos ni rutinas, con otras formas de conocimiento (cosmovisiones, filosofías, saberes populares-cultivados-místicos-artísticoscientíficos-humanísticos, entre otros) para ampliar la pluri-comprensión de mundos desde una "multi-inter-trans-epistemología” que permita "multiinter-trans-interpretaciones” para ampliar nuestra comprensión de la complejidad del todo azaroso, incierto, caórdico, cambiante, que es el mundo.

\section{La Onto-trans-epistemología Compleja de la Creactividad} enfatiza en la praxis de la Complejidad a partir de vivenciar un proceso de fecundación-gestación-florecimiento-apreciación de un enfoque policontextual relacionado con la Creatividad como raíz de la Sociedad del Conocimiento y las formas de conocer (nos) y re-crear (nos).

La praxis habita en la teoría. Ambas coexisten teórico-prácticamente sin reducirse la una en/a la otra; por el contrario, teoría y praxis se fomentan y amplían desarrollando sus resortes entrelazadores "multi-intertrans-epistémicos” y “multi-inter-trans-prácticos” a partir de detonadores lúdico-didácticos que hemos denominado Co-osadías Caordiscientes para “darse a luz” Creactores.

El concepto de Multi-praxis, refiere a la aplicación de diversas dinámicas y juegos de forma lineal, progresiva y sistemática. Por su parte la Interpraxis, se expresa en religaciones lúdico-andragógicas apoyadas en la combinación y/o mezcla de una serie de ejercicios que van componiendo de manera creativa nuevas estrategias didácticas tomando como base a los elementos existentes. Y la Trans-praxis, por su parte, trasgrede lo estipulado, trasciende lo planificado, emerge lúdico-didácticamente como necesidad del instante, como espontaneidad innovactiva que re-direcciona hacia nuevos horizontes "procursionando" la aplicabilidad de dicha teoría-praxis y fertilizando

ocultos, creativos, expresiones invisibles que habitan los vocablos camuflajeadas por las convenciones y los prejuicios personales/sociales/disciplinares. El concepto de desempalabrar inspira el ir al encuentro con locuciones preñadas de vida, de palabras dentro de palabras que al re-empalabrarlas des-ocultan nuevos términos, hacen patente neologismos para re-significar el mundo. Empalabrando nuevas voces, se abren distintos caminos inteligibles que nos facilitan y vinculan con las tramas, senderos, giros y metáforas del Pensamiento Complejo, desempalabrando y reempalabrando locuciones, fundamos territorios para las filosofías, ciencias y artes emergentes, pues las palabras son el pulso más notorio de lo humano, las palabras son al hombre lo que el big - bang al universo. 
creactivamente sus nuevos nortes de acción y creacción.

Desde la Creactividad en sí misma se abre una posibilidad para el desarrollo de una praxis andragógica en/desde/con y para la Complejidad, con sus dinámicas lúdicas, cogno-vivenciales, cogno-meta-conscientes expresadas en Co-osadías Caordiscientes, entendidas como transjuegos o estrategias meta-conscientes para el co-aprendizaje y co-des-aprendizaje creactivo, metaforador y paradojador, capaces de recrear el cognoscimiento en una especie de co-auto-eco-innovación trans-cognitiva, en y desde una Creactividad-Compleja y una Complejidad-Creactiva, utilizando instrumentos como, por ejemplo, la holontotelarañización, especie de ensamblaje trans-multi-epistémico trans-hermenéutico que se abre a pluriposibilidades, pluri-mundos y multiversos que van trans-ocurriendo en el mismo instante del devenir.

Ahora bien... ¿Cómo es que toda esta multi-inter-trans valoración e interpretación puede ser reconocible? o más bien: ¿Re-cogno-asible? El concepto emergente, la idea-fuerza, es el "instante creactivo"

Los instantes de la creacción, los instantes creactivos, los instantes de la Creactividad, son como centelleos que nos permiten apreciar lo implícito, lo virtual, lo omiso y silencioso que albergamos en nuestro Ser. En y desde ellos el potencial mismo emerge como fuente activando/accionando ontológicamente el poder creactor, que se manifiesta y nos sorprende “dándonos a luz” en tanto seres capaces de creacción; trascendiendo(nos) por un instante en/de nuestras propias sujeciones, nuestras propias cadenas heredadas (epistémicas, culturales, religiosas, políticas, entre otras), inconscientes o no; haciendo explícita nuestra propia "luz", nuestro propio brillo capaz de alumbrar inmanente/trascendentemente nuestras sombras ${ }^{10}$ que, al verse descubiertas, se disipan en oportunidades de manifestación de la totalidad que vamos siendo, totalidad de divergencias y convergencias, de talentos y dones, de inteligencias, consciencias y competencias que somos siendo en cada instante, consciente o no, a la vez.

Para Heidegger en 'Ser y Tiempo’, el instante es:

... ‘el presente auténtico’... oponiéndolo al ahora que es el presente impropio de la vida cotidiana. El ahora es la pre-

\footnotetext{
${ }^{10}$ Las sombras, que paradójicamente sólo ocurren cuando hay luz, a manera de metáfora, representan en este contexto, nuestras limitaciones, obstáculos, supersticiones, reticencias, impedimentos (físicos/psíquicos/socio-culturales), sujeciones y encadenamientos heredados, condicionamientos (sociales/religiosos/filosóficos/

políticos), tabúes, barreras asumidas por inmadurez (emocional/interpersonal/espiritual) inconscientes (o no), entre otras. Sombras que, paradójicamente de nuevo, se pueden disipar "iluminándolas" (con las distintas formas, técnicas, métodos que las personas elijan). Las de la onto-trans-epistemología emergen "cogno-vivenciándolas” desde/con las coosadías caordiscientes, en/desde la "cogno-meta-consciencia”, con la "refle-acción creactiva”, con/desde la "multi-inter-trans-hermenéutica”, entre otras prácticas posibles.
} 
sentación de las cosas a las que se dirige la atención cotidiana del hombre; el instante es la decisión anticipadora de la muerte, esto es la nada de la existencia (citado en Abbagnano y Fornero, 2004:606)

Y las co-osadías caordiscientes precisamente pretenden/detonan y en los talleres desarrollados como praxis pretendieron y detonaron - ontotrans-epistémicamente, la posibilidad/potencialidad de caordiscientizar algo y/o alguien ¿Cómo? sería la pregunta... entre otras respuestas posibles elegimos decir que des-significando(se), re-significando(se), desaprendiendo(se), re-aprendiendo(se), metaforando(se), paradojando(se), deconstruyendo(se), multi-inter-trans-hermeneutizando(se), es decir: caordiscientizando(se).

Todo ello implica la muerte, en un instante, del Sí mismo, de la totalidad del "yo soy” que ha venido siendo; la decisión osada, co-osada y cogno-consciente, metafórico/paradójica de matar(se), de matar a ese "yo soy” convenido al que se está habituado, ese que se identifica más con el afuera, con lo cotidiano y ordinario, con las cosas externas de sí y del mundo, que se identifica más con lo que tiene/posee que con lo que es; una muerte entendida como cambio/transformación/mutación; muerte como decisión cogno-consciente de transitar el camino “caósmico” (Guattari,1996) o "caórdico" (Andrade y Pereira, 2006) que va del cosmos (lo conocido) al caos (lo desconocido) de uno mismo como Ser... para re-ordenar(se) en cada instante posible/potencial de la existencia; una muerte onto-transepistémica creactiva compleja indispensable para “darse a luz” Creactores porque es muerte una y cada vez de las sombras y cadenas heredadas; muerte en la oportunidad del instante de irse "dando a luz" como posibilidad/potencialidad creactiva de su yo-múltiple siendo; una muerte anticipada para “fenixientemente” re-nacer(se), re-iluminar(se), re-encender(se) inmanente y trascendentemente en un instante creactivo, un instante de creacción y trascendencia de sí mismo, de los propios límites y prejuicios que nos/le/ los igualan banalizando y esclavizando nuestra existencia, adormeciendo la autenticidad originaria y propia de cada uno ${ }^{11}$. Donde la unidad es cada emergencia ontológica-fenomenológica del "darse a luz" Creactores y la totalidad es el devenir fáctico y hermenéutico de la Onto-trans-epistemología Compleja de la Creactividad.

En la Creactividad de la Complejidad donde todo “es-siendo” permanentemente paradoja, incertidumbre caordisciente, cambio y fluidez multi-dimensional, aventura y desafío multi-inter-trans-conceptual en el devenir, los conceptos son vistos como posibilidad/potencialidad y deseo-

${ }^{11}$ La cual puede ser "re-apropiada", no en la experiencia del devenir horizontal que nos empuja adormilando entrópicamente la consciencia de sí, sino en la "cogno-vivencia del instante creactivo" en el que "soy", del "yo-múltiple- siendo", donde cada quien puede, potencialmente, "re-cogno-ser(se), creactivar(se)”, es decir... “darse a luz” Creactor/ Creactríz. 
fuerza aprovechables por los Creactores que los caordiscientizan, ya que, cogno-conscientemente, saben que en la Onto-trans-epistemología Compleja de la Creactividad, los conceptos son, al mismo tiempo, tanto apertura luminosa que motiva, como enclaustre histórico en el sentido de la tradición.

Los Creactores comprehenden que los conceptos siempre son susceptibles de ser migrados, metaforeados, exprimidos y recoloreados, que también pueden ser asumidos desde su destino ya que los mismos significan/des-significan/re-significan mundos, pueden ser apropiados desde la viabilidad que nos permite conceptuarlos/des-conceptuarlos/re-conceptuarlos, con lo que se pueden construir/de-construir/re-construir y teorizar/desteorizar/re-teorizar, en un proceso des-limitador/re-limitador de todo cuanto nos importa: la Vida. Creactores en tanto un "Sí mismo- siendo" Libertad, diversidad de mundos, multiverso abierto a la "felicidad creactiva".

La Onto-trans-epistemología Compleja de la Creactividad se reconoce "creactora de realidades", de horizontes de comprensión, de valores vitalistas capaces de trascender en y desde la inmanencia con la "valentía-andragógica” de los Creactores, ya que el Creactor y la Creactriz logran "darse a luz" "multi-inter-trans-hermeneutizando(se)" cada uno a sí mismo, de-construyendo sus propios límites.

\section{Huellas polifónicas}

La huellas polifónicas entendiendo nuestra búsqueda como cruces de caminos, como un atrevernos a seguir imaginando rumbos posibles, territorializaciones y desterritorializaciones que hagan de la Educación Superior, del aprendizaje, desaprendizajes y re-aprendizajes, procesos vivos, onto-trans-epistémicos y caordiscientes que permitan vivenciar en las ARCAS, una praxis de la Complejidad, en un continuum de desempalabramientos caórdicos que detonen la construcción y deconstrucción de nuevas significaciones en el camino del Ser, del Conocer, del Hacer y del Convivir creactivos. 


\section{Bibliografía}

Abbagnano y Fornero (2004), Diccionario de filosofía, Fondo de Cultura Económica, Ciudad de México.

Andrade, R. (2003), Aprender a desaprender. Juegos dialógicos de desaprendizaje cognoconsciente, Tesis Doctoral en Educación, Universidad Interamericana de Educación a Distancia de Panamá (UNIEDPA). Idem. (2004), "Hacia un desaprendizaje consciente”, Fermentum, Año 14, No 39 , Mérida.

Andrade, R. y Pereira, L. (2006), “Fárbices caórdicas. Nuevos lenguajes organizacionales en la era de la complejidad” en Polis, Revista de la Universidad Bolivariana, Volumen 5 N 15, Santiago.

Barbier, R. (1997), El educador como "passeur de sens". [Documento en línea]. Ponencia presentada en el Congreso Internacional ¿Cuál Universidad para Mañana? Hacia una evolución transdisciplinaria de la universidad, Suiza. (N. Núñez y G. Dentón, Trads.). Disponible:_http://www.barbierrd.nom.fr/EducatorRB.PDF [Consulta: 2005, Agosto 27]

Copleston, F. (2004), Historia de la filosofía. Barcelona: Ariel. Quinta Edición. Vols. 8, 9.

Cyrulnik, B.; Morin, E. (2005), Diálogos sobre la naturaleza humana, Paidós, Barcelona.

Deleuze,G.; Guattari, F. (2003), Rizoma. Introducción, Pre-textos, Valencia.

Delors, J. (1996), La educación encierra un tesoro, Santillana y Ediciones UNESCO, Madrid.

Derrida, J. (2001), „Palabra!, Trotta, Madrid.

Dilthey, W. (1960), La esencia de la filosofía, Losada S.A., Buenos Aires.

Drucker, P. (1968), The age of discontiuity. A guidelines to our changing society, Harper Collins, New York.

Fainholc, B. (2005), ¿Educar para qué tipo de sociedad del conocimiento? en Educar [Revista en línea]. Disponible: http://portal.educ.ar/debates/ educacionytic/debate/educar-para-que-tipo-de-sociedad-delconocimiento.php [Consulta: 2005, Junio 28]

Flores, L. (2006), Reflexiones fenomenológicas sobre la subjetividad humana: Hacia una recuperación compleja de la conciencia encarnada, en III Seminario Bienal Internacional acerca de las Implicaciones Filosóficas, Epistemológicas y Metodológicas de la Teoría de la Complejidad. Complejidad La Habana 2006, organizado por la Cátedra para el Estudio de la 
Complejidad del Instituto de Filosofía de La Habana y Co-Auspiciado por el Ministerio de Ciencia, Tecnología y Medio Ambiente (CITMA) y la Academia de Ciencias de Cuba, La Habana del 09 al 12 de enero de 2006. Palacio de Las Convenciones de La Habana, Cuba.

Foucault, M. (1979), Las palabras y las cosas, Siglo XXI, Ciudad de México.

Guattari, F. (1996), Caosmosis, Ediciones Manantial, Buenos Aires.

Heidegger, M. (1987), De camino al habla, Odós, Barcelona.

Idem (1997), Ser y tiempo. (Trad. Rivera, J.), Editorial Universitaria, Santiago.

Martínez Andrade, D. (2009), Onto-trans-epistemología compleja de la creactividad. Co-osadías caordiscientes para "darse a luz" creactores, Tesis Doctoral en Educación, Universidad Interamericana de Educación a

Distancia de Panamá (UNIEDPA).

Méndez, R. (2009), Principios gnoseológicos del co-entrelazamiento de aprendizajes en el contexto de la complejidad. Hacia un Aula Rizomática Co-entrelazadora de Aprendizajes (ARCA) desde una perspectiva Cognovivencial, Tesis Doctoral en Educación, Universidad Interamericana de Educación a Distancia de Panamá (UNIEDPA).

Morin, E. (1997), Introducción al pensamiento complejo (M. Pakman, Trad.), Gedisa, Barcelona.

Najmanovich, D. (2006), El desafío de la complejidad, en III Seminario Bienal Internacional acerca de las Implicaciones Filosóficas, Epistemológicas y Metodológicas de la Teoría de la Complejidad. Complejidad La Habana 2006, organizado por la Cátedra para el Estudio de la Complejidad del Instituto de Filosofía de La Habana y Co-Auspiciado por el Ministerio de Ciencia, Tecnología y Medio Ambiente (CITMA) y la Academia de Ciencias de Cuba, La Habana del 09 al 12 de enero de 2006. Palacio de Las Convenciones de La Habana, Cuba.

Idem (2008), Mirar con nuevos ojos: nuevos paradigmas en la ciencia y pensamiento complejo, Biblos, Buenos Aires.

Schopenhauer, A. (1818), El mundo como voluntad de representación, en Atlas Universal de Filosofía, Océano, Barcelona.

Sheldrake, R.; McKenna, T. y Abraham, R. (2005), Caos creatividad y consciencia cósmica, Eliago, Castellón. 